\title{
Prognostic Significance of Focal Adhesion Kinase in Node-Negative Breast Cancer
}

\author{
Katrin Almstedt ${ }^{a} \quad$ Isabel Sicking $^{a} \quad$ Marco J. Battista ${ }^{a}$ Shangou Huangfu ${ }^{a}$ \\ Anne-Sophie Heimes ${ }^{a}$ Veronika Weyer-Elberich ${ }^{b}$ Annette Hasenburg ${ }^{a}$ Marcus Schmidt ${ }^{a}$

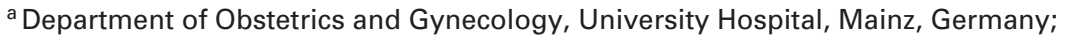 \\ ${ }^{\mathrm{b}}$ Institute of Medical Biostatistics, Epidemiology and Informatics (IMBEI), Johannes Gutenberg University, Mainz, Germany
}

\section{Keywords}

Focal adhesion kinase · Breast cancer · Node-negative · Prognosis · Protein

\section{Summary}

Background: Focal adhesion kinase (FAK) is a cytoplasmic tyrosine kinase that plays an important role as a mediator of cell migration, invasion, proliferation and survival. Conflicting results for the prognostic role of FAK in breast cancer $(\mathrm{BC})$ prompted us to determine its impact. Methods: Patients with node-negative BC entered this retrospective study. FAK expression was determined by immunohistochemistry ( $n=335)$. The prognostic impact of FAK was examined with Cox regression analyses and Kaplan-Meier estimation in the whole cohort as well as in different molecular subtypes. Results: 151 (45.1\%) had a FAK-positive BC. In univariate analyses, FAK expression showed a significant impact for shorter disease-free survival (DFS) (hazard ratio (HR) 1.54, 95\% confidence interval (Cl) 1.04-2.28, $\mathrm{p}=0.030$ ) but not for metastasisfree survival and overall survival. Significant prognostic relevance for DFS (HR 1.76, 95\% Cl 1.05-2.97, $p=0.033$ ) was observed in particular in estrogen receptor-positive HER2-negative BC patients, most notably in luminal Blike tumors (HR 2.32, $\mathrm{Cl} 1.20-4.48, \mathrm{p}=0.012$ ). However, FAK lost its prognostic impact in multivariate Cox regression analysis. Conclusion: FAK was associated with impaired DFS in univariate analysis. Prognostic relevance for DFS was most pronounced in luminal B-like BC. However, FAK expression was not associated with an independent impact on survival for $B C$ in multivariate analysis.

(c) 2017 S. Karger GmbH, Freiburg

\section{Introduction}

Focal adhesion kinase (FAK) is a non-receptor-type tyrosine kinase that plays a crucial role in integrin-mediated cellular interaction between the intracellular cytoskeleton and the extracellular matrix, as well as in growth factor- and cytokine receptor-mediated pathways [1]. As a part of focal adhesions, FAK is essential for tumorigenesis by regulating adhesion-independent cell growth, proliferation, survival, migration, invasion and angiogenesis [2]. Several studies have shown an association between high expression of FAK and/or fak gene amplification and progression to an invasive cell phenotype, including breast cancer (BC) $[3,4]$, whereas in benign breast tissue FAK expression is rarely detectable [5]. Cance and colleagues $[5,6]$ showed that FAK was upregulated in BC. Because of moderate levels for FAK expression in pre-invasive ductal carcinoma in situ cells, the authors concluded that high expression of FAK may be an early event in carcinogenesis of BC. Regarding the prognostic role of FAK in BC, studies have conflicting results. Other studies describe FAK associated with suppression of mammary tumorigenesis, and that silencing FAK reduces the pool of cancer stem cells in mice [2] and reduces mammary tumor formation [7].

The aim of the current study was to examine the prognostic impact of FAK in an untreated cohort of lymph node-negative BC patients with a focus on different molecular subtypes.

\section{Patients and Methods}

\section{Patient Cohorts}

To determine the prognostic impact of FAK we selected patients with nodenegative BC treated between 1985 and 2000 at the University Hospital Mainz, who did not receive any adjuvant treatment modalities after tumor resection. Patients with available tumor samples and consistent information on follow-up entered this retrospective explorative study. The initial study cohort consisted of 410 consecutive lymph node-negative BC patients. Tumor tissue for FAK im-

\section{KARGER}

(C) 2017 S. Karger GmbH, Freiburg

Fax +497614520714 

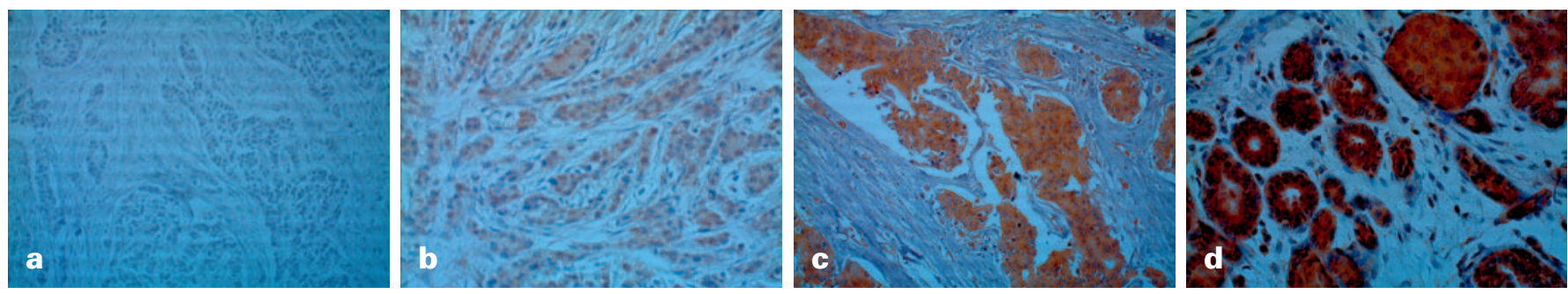

Fig. 1. Representative examples of FAK immunostaining in breast cancer. a Negative FAK staining intensity (FAK score: 0 ). b Weak FAK staining intensity (FAK score: 1+). c Moderate FAK staining intensity (FAK score: $2+$ ). d Strong FAK staining intensity (FAK score: $3+$ ).

munohistochemistry was available for 335 patients. Adequate follow-up information of all patients was available. Patients were treated either with modified radical mastectomy (44\%) or breast-conserving surgery followed by irradiation (56\%). All patients received a complete axillary dissection. They did not receive any systemic therapy in the adjuvant setting. Patient and tumor characteristics were collected retrospectively in a previously published BC database [8]. Breast cancer subtypes were classified as luminal A-like $\left(\mathrm{ER}^{+}\right.$and/or $\mathrm{PR}^{+}, \mathrm{HER} 2^{-}$, Ki-67 $\leq 20 \%$ ), luminal B-like (ER ${ }^{+}$and/or PR ${ }^{+}$, HER2 ${ }^{-}, \mathrm{Ki}-67>20 \%$ ), HER2positive $\left(\mathrm{HER}^{+}\right)$, and triple-negative $\left(\mathrm{ER}^{-}, \mathrm{PR}^{-}, \mathrm{HER} 2^{-}\right)$.

The study was approved by the ethical review board of the medical association of Rhineland-Palatinate. The study was conducted according to the reporting recommendations for tumor marker reporting studies [9].

\section{Immunohistochemistry}

Immunohistochemical staining for FAK was performed according to standard procedures. Briefly, $4-\mu \mathrm{m}$-thick serial section of formalin-fixed and paraffin-embedded (FFPE) tumor tissue slides were incubated with the primary antibody in a dilution of 1:100 (rabbit anti-human-FAK antibody, Cell Signaling Technology), followed by polymeric biotin-free visualization system (Envision $^{\mathrm{TM}}$; DAKO Diagnostic Company, Hamburg, Germany). Finally, the sections were counterstained with Mayer's hematoxylin solution (Sigma-Aldrich ${ }^{\circledR}$ ). We used a BC sample strongly positive for FAK as a positive control. Negative controls were prepared by substituting normal mouse serum for primary antibody, and no detectable staining was evident. FAK immunostaining was evaluated by 2 of the authors (S.H. and M.S.) experienced in histological and immunohistochemical diagnostics unaware of the clinical outcome. Since evaluation of FAK expression is not yet standardized, we used the scoring system for estrogen receptor (ER) and progesterone receptor (PR) in accordance to Remmele and Stegner [10]. It was defined by the product of a proportion $(0$, none; $1,<10 \% ; 2$, $10-50 \% ; 3,51-80 \% ; 4,81-100 \%)$ and an intensity score ( 0 , no staining; 1 , weak; 2 , moderate; 3 , strong) [10]. The median was used to dichotomize the samples into FAK positive (high expression) and negative (low expression) tumors.

\section{Statistical Analyses}

Statistical analysis was performed with the SPSS statistical software program, version 22.0 (SPSS Inc., Chicago, IL, USA). Multivariate analyses were conducted using Cox regression analysis to evaluate the association between FAK expression (FAK positive vs. negative) in the BC samples and established prognostic factors such as age at diagnosis ( $<50$ years vs. $\geq 50$ years), tumor size (pT1 vs. $\geq$ pT2), histological grade (grade 1 and 2 vs. grade 3 ), hormone receptor status (ER or PR immunoreactive score (IRS) 1 or higher vs. IRS 0 ) and HER2 status (HER2 $3+$ or HER2 2+ and Fish positive vs. HER2 0, 1+ and HER2 2+ and Fish negative). Ki-67 was divided in low ( $\leq 20 \%$ positive cells) and high $(>20 \%)$. Death from $\mathrm{BC}$ or unrelated to $\mathrm{BC}$, and metastasis or local relapse were documented. Metastasis-free survival (MFS) was defined as the time between surgery and diagnosis of metastasis. Disease-free survival (DFS) was defined as the time between the date of surgery and the date of loco-regional or metastatic recurrence, BC related death or lost follow-up. Overall survival (OS) was defined as time between the date of surgery and the date of death through any cause. The correlation between FAK and clinico-pathological parameters was assessed using chi-square statistics. Univariate and multivariate Cox regression analyses were performed. Survival rates were calculated by the Kaplan-Meier method and survival times were compared using the log-rank test. All $\mathrm{p}$ values were 2 -sided. A p value of $<0.05$ was considered statistically significant. Since no correction for multiple testing was performed results are interpreted as explorative.

\section{Results}

\section{Patient Cohorts}

We screened 410 consecutive patients with lymph node-negative BC. For 335 patients FFPE tissue was available and these entered the study for FAK immunohistochemistry. The mean followup time for the entire cohort was 183 months (range 0-348 months). 63 patients (18.8\%) died from BC, 47 (14.0\%) died from causes unrelated to $\mathrm{BC}$ and 211 patients $(63.0 \%)$ were alive at the date of last follow-up; 14 patients (4.2\%) died of unknown causes. 43 patients (12.8\%) suffered from local recurrence and 75 (22.4\%) developed distant metastasis. Patients' demographic data are summarized in table 1.

\section{Immunohistochemistry}

Representative pictures of FAK immunostaining are shown in figure 1 and present a mainly cytoplasmic and occasionally membranous staining. As described before, tissue samples were divided into FAK high/positive (multiplication product of $\geq 6,45.1 \%$, $\mathrm{n}=151$ ) and low/negative (all other cases, $54.9 \%, \mathrm{n}=184$ ) expressing samples (table 1).

\section{Correlation of FAK and Clinico-Pathological Variables}

When we performed correlation analyses using chi-square tests with FAK and the categorized clinico-pathological variables age, tumor size, histological grade of differentiation, ER, PR and HER2, only ER showed a significant correlation with FAK ( $p=0.043)$.

\section{Association of FAK with DFS}

High expression of FAK was associated with better DFS (hazard ratio (HR) 1.54, 95\% confidence interval (CI) 1.04-2.28, p = 0.030) (table 2). Kaplan-Meier plots revealed a 76.9\% 5-year DFS rate for FAK-positive and an 84.5\% 5-year DFS rate for FAK-negative patients ( $\mathrm{p}=0.028$ ) (fig. 2). In addition to FAK tumor size (HR 1.67, 95\% CI 1.13-2.47, $\mathrm{p}=0.010$ ), grading (HR 3.10, 95\% CI 2.08-4.63, $\mathrm{p}=<0.001$ ), ER status (HR 0.58, 95\% CI 0.38-0.88, $\mathrm{p}=0.011$ ), HER2 status (HR 2.48, 95\% CI 1.54-3.98, $\mathrm{p}=<0.002$ ) and Ki-67 (HR 1.85, 95\% CI 1.23-2.77, $\mathrm{p}=0.003$ ) showed significant influence in univariate analysis (table 2). However, in multivariate anal- 
Table 1. Patients' characteristics in the entire cohort of node-negative breast cancer patients $(\mathrm{n}=335)$

\begin{tabular}{|c|c|c|}
\hline \multirow{3}{*}{$\begin{array}{l}\text { Median follow-up, month (Max) } \\
\text { Median age, years (range) }\end{array}$} & \multirow{2}{*}{\multicolumn{2}{|c|}{$\begin{array}{l}183(348) \\
58(32-90)\end{array}$}} \\
\hline & & \\
\hline & $\mathrm{n}$ & $\%$ \\
\hline \multicolumn{3}{|l|}{ Age } \\
\hline$<50$ years & 94 & 28.1 \\
\hline$\geq 50$ years & 241 & 71.9 \\
\hline pT stage & 0 & 0 \\
\hline \multicolumn{3}{|l|}{ pTis } \\
\hline pT1 & 213 & 63.6 \\
\hline pT1a & 20 & 6.0 \\
\hline pT1b & 56 & 16.7 \\
\hline pT1c & 137 & 40.9 \\
\hline pT2 & 118 & 35.2 \\
\hline pT3 & 4 & 1.2 \\
\hline pT4 & 0 & 0 \\
\hline \multicolumn{3}{|l|}{ Histological grade } \\
\hline 1 & 79 & 23.6 \\
\hline 2 & 183 & 54.6 \\
\hline 3 & 73 & 21.8 \\
\hline \multicolumn{3}{|l|}{ ER status } \\
\hline Negative & 78 & 23.3 \\
\hline Positive & 257 & 76.7 \\
\hline \multicolumn{3}{|l|}{ PR status } \\
\hline Negative & 104 & 31.0 \\
\hline Positive & 231 & 69.0 \\
\hline \multicolumn{3}{|l|}{ HER2 status } \\
\hline Negative & 293 & 87.5 \\
\hline Positive & 42 & 12.5 \\
\hline 0 & 228 & 68.1 \\
\hline 1 & 58 & 17.3 \\
\hline 2 & 9 & 2.7 \\
\hline FISH negative & 7 & 2.1 \\
\hline FISH positive & 2 & 0.6 \\
\hline 3 & 40 & 11.9 \\
\hline \multicolumn{3}{|l|}{ Ki-67 expression $(\mathrm{n}=323)$} \\
\hline Low & 231 & 71.5 \\
\hline High & 92 & 28.5 \\
\hline Missing & 12 & \\
\hline \multicolumn{3}{|l|}{ FAK staining intensity } \\
\hline Negative (0) & 22 & 6.6 \\
\hline Weak (1) & 124 & 37.0 \\
\hline Moderate (2) & 114 & 34.0 \\
\hline Strong (3) & 75 & 22.4 \\
\hline
\end{tabular}

ysis, FAK failed to reach significance for DFS (table 2). Only histological grade of differentiation (HR 2.63, 95\% CI 1.56-4.42, $\mathrm{p}=<0.001$ ) and HER2 status (HR 1.99, 95\% CI 1.21-3.28, $\mathrm{p}=0.007)$ retained an independent influence for DFS.

FAK was not associated with either MFS (HR 1.34, 95\% CI $0.85-2.11, \mathrm{p}=0.206$ ) or OS (HR 1.18, 95\% Cl 0.83-1.67 p = 0.369). Concerning MFS, prognostic significance was obtained for tumor size (HR 1.69, 95\% CI 1.08-2.67, p = 0.023), grading (HR 3.73, 95\% CI $2.37-5.88, \mathrm{p}=<0.001$ ), HER2 status (HR 2.95, 95\% CI $1.75-$ $4.96, \mathrm{p}=<0.002)$ and Ki-67 (HR 1.83, 95\% CI 1.15-2.92, $\mathrm{p}=0.011)$. For OS, influence was shown for age at diagnosis (HR 2.14, 95\% CI
Table 1. Continued

\begin{tabular}{lcc}
\hline Median follow-up, month (Max) & \multicolumn{2}{l}{$183(348)$} \\
Median age, years (range) & $58(32-90)$ & \\
\cline { 2 - 3 } & $\mathrm{n}$ & \\
\cline { 2 - 2 } FAK positive cells, \% & 22 & 6.6 \\
$\quad 0$ & 23 & 6.9 \\
1 & 55 & 16.4 \\
2 & 58 & 17.3 \\
3 & 177 & 52.8 \\
4 & & \\
FAK expression IHC & 184 & 54.9 \\
$\quad$ Low & 151 & 45.1 \\
$\quad$ High & & \\
Death & 63 & 18.8 \\
$\quad$ Due to breast cancer & 47 & 14.0 \\
$\quad$ Unrelated to breast cancer & 14 & 4.2 \\
$\quad$ Unknown causes & 211 & 63.0 \\
Surviving & & \\
Local recurrence & 43 & 12.8 \\
$\quad$ Yes & 292 & 87.2 \\
$\quad$ No & & \\
Metastasis & 75 & 77.6 \\
$\quad$ Yes & 260 & \\
No & & \\
\hline
\end{tabular}

$\mathrm{ER}=$ Estrogen receptor; $\mathrm{PR}=$ progesterone receptor; $\mathrm{FAK}=$ focal adhesion kinase; IHC = immunohistochemistry.

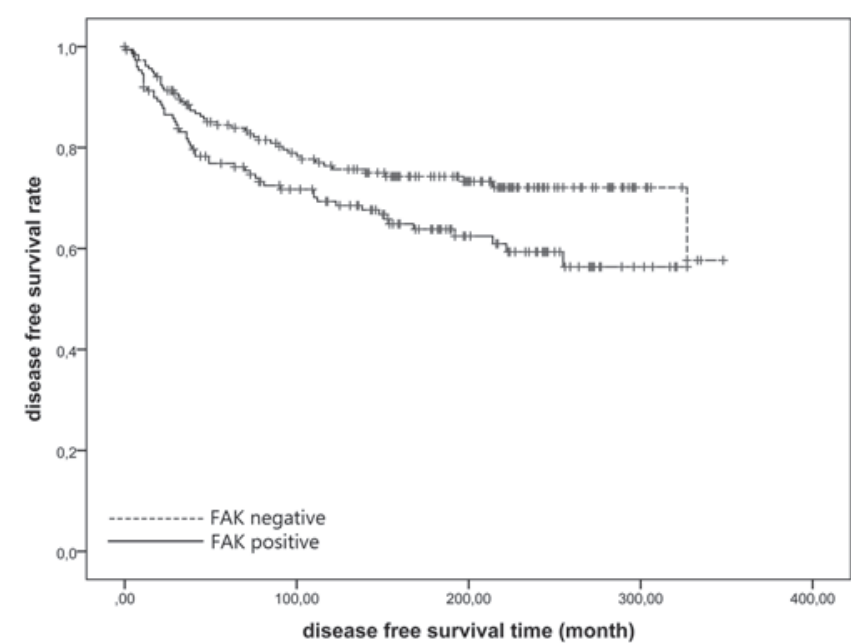

5 years rate $\begin{array}{ll}\text { FAK negative } & 84.5 \% \\ \text { FAK positive } & 76.9 \%\end{array}$

logRank test: 0.028

Fig. 2. Association of focal adhesion kinase (FAK) expression with diseasefree survival (DFS) in the entire cohort $(n=335)$.

1.37-3.36, $\mathrm{p}=0.001$ ), tumor size (HR 1.43, 95\% CI 1.00-2.03, $\mathrm{p}=0.050)$, grading (HR 2.32, 95\% CI 1.60-3.36, $\mathrm{p}=<0.001)$, HER2 status (HR 1.75, 95\% CI 1.10-2.77, p = 0.018) and Ki-67 (HR 1.56, $95 \%$ CI 1.07-2.26, $\mathrm{p}=0.020)$. Kaplan Meier analyses failed to show a significant difference for MFS (74.8 vs. $79.9 \%, \mathrm{p}=0.204)$ and OS (60.3 vs. $65.2 \%, \mathrm{p}=0.367$ ) 
Table 2. Association of FAK immunostaining (positive (6-12) vs. negative (0-4)) with breast cancer-specific DFS in the entire cohort of node-negative breast cancer patients $(\mathrm{n}=335)$

\begin{tabular}{lccc}
\hline Prognostic factor & p value & HR & 95\% CI \\
\hline Univariate Cox analysis & & & \\
FAK IHC status (positive vs. negative) & 0.030 & 1.54 & $1.04-2.28$ \\
Age ( $\geq 50$ vs. $<50$ years) & 0.264 & 0.79 & $0.52-1.20$ \\
pT stage ( $>2$ vs. $<2$ cm) & 0.010 & 1.67 & $1.13-2.47$ \\
Histological grade (grade 3 vs. grade 1 and 2) & $<0.001$ & 3.10 & $2.08-4.63$ \\
ER (positive vs. negative) & 0.011 & 0.58 & $0.38-0.88$ \\
PR (positive vs. negative) & 0.361 & 0.83 & $0.55-1.24$ \\
HER2 status (positive vs. negative) & $<0.002$ & 2.48 & $1.54-3.98$ \\
Ki-67 (high vs. low) & 0.003 & 1.85 & $1.23-2.77$ \\
Multivariate Cox analysis & & & \\
FAK IHC status (positive vs. negative) & 0.244 & 1.28 & $0.85-1.92$ \\
pT stage (>2 vs. $<2$ cm) & 0.212 & 1.30 & $0.86-1.96$ \\
Histological grade (grade 3 vs. grade 1 and 2) & $<0.001$ & 2.63 & $1.56-4.42$ \\
ER (positive vs. negative) & 0.460 & 1.20 & $0.74-1.94$ \\
HER2 status (positive vs. negative) & 0.007 & 1.99 & $1.21-3.28$ \\
Ki-67 (high vs. low) & 0.854 & 1.05 & $0.65-1.70$ \\
\hline
\end{tabular}

FAK = Focal adhesion kinase; DFS = disease-free survival; $\mathrm{IHC}$ = immunohistochemistry; $\mathrm{ER}=$ estrogen receptor; $\mathrm{PR}=$ progesterone receptor; $\mathrm{HR}=$ hazard ratio; $\mathrm{CI}=$ confidence interval.

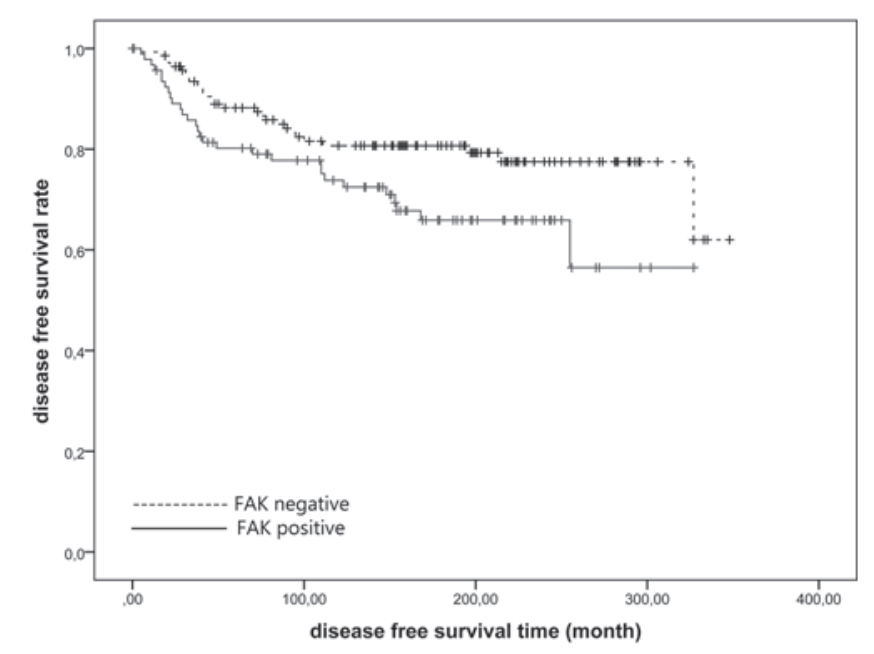

5 years rate

Fig. 3. Association of FAK expression with DFS in the $\mathrm{ER}^{+} \mathrm{HER} 2^{-}$cohort $(\mathrm{n}=334)$.

\section{Subgroup Analyses}

In a next step, we examined the prognostic impact of FAK within different molecular subtypes. Considering the different molecular subtypes, the prognostic effect for DSF was most pronounced in $\mathrm{ER}^{+}$HER2- ${ }^{-}$carcinomas (HR 1.76, 95\% CI 1.05-2.97, $\mathrm{p}=0.033$ ). Kaplan-Meier plots showed an $80.2 \% 5$-year DFS rate for FAK-positive and an 88.2\% 5-year DFS rate for FAK-negative patients ( $\mathrm{p}=0.031$ ) (fig. 3 ). The association of FAK with DFS was most pronounced in luminal B-like BC (HR 2.32, 95\% CI 1.20$4.48, \mathrm{p}=0.012$ ). FAK expression failed to show a significant association with all other endpoints in any other subtypes of $\mathrm{BC}$ (table 3).
Table 3. Association of FAK immunostaining (positive (6-12) vs. negative $(0-4)$ ) with breast cancer-specific prognostic endpoints of node-negative breast cancer patients within different molecular subtypes

\begin{tabular}{|c|c|c|c|}
\hline Prognostic endpoint & $\mathrm{p}$ value & $\mathrm{HR}$ & $95 \% \mathrm{CI}$ \\
\hline \multicolumn{4}{|l|}{$\mathrm{ER}^{+} \operatorname{HER}^{-}(\mathrm{n}=234)$} \\
\hline DFS & 0.033 & 1.76 & $1.046-2.966$ \\
\hline MFS & 0.188 & 1.509 & $0.818-2.784$ \\
\hline OS & 0.379 & 1.229 & $0.777-1.944$ \\
\hline \multicolumn{4}{|l|}{ ER $^{-}$HER2- $(n=59)$} \\
\hline DFS & 0.866 & 0.930 & $0.402-2.154$ \\
\hline MFS & 0.470 & 0.688 & $0.249-1.897$ \\
\hline OS & 0.792 & 0.905 & $0.430-1.903$ \\
\hline \multicolumn{4}{|l|}{$\mathrm{ER}^{-} \mathrm{HER}^{+}(\mathrm{n}=42)$} \\
\hline DFS & 0.754 & 1.145 & $0.489-2.683$ \\
\hline MFS & 0.693 & 1.202 & $0.482-2.998$ \\
\hline OS & 0.953 & 0.975 & $0.421-2.260$ \\
\hline
\end{tabular}

DFS $=$ Disease-free survival; MFS = metastasis-free survival; OS $=$ overall sur vival.

\section{Discussion}

Prognostic factors play a crucial role in the adequate treatment of patients with early BC. In our retrospective study, expression of FAK was associated with poor DFS in node-negative BC. The prognostic significance of FAK was shown for DFS in univariate analysis, especially in $\mathrm{ER}^{+} \mathrm{BC}$. This effect was most pronounced in luminal B-like tumors. However, caution in interpreting our finding is necessary due to the very small sample size of this subgroup $(\mathrm{n}=21)$. In multivariate analyses, FAK failed to show any association with survival. We did not differentiate between phosphorylated/activated and inactivated FAK proteins. Madan et al. [11] performed this differentiation, but the hypothesis that activated FAK (FAK $\mathrm{pY}^{397}$, main autophosphorylation site) was associated with 
tumor invasiveness was not verified. In addition to the biological aspects, there is a methodical challenge in evaluating the influence of FAK in prognosis for $\mathrm{BC}$ as there is no standardized cut-off value. In our study, based on results of preliminary data, we chose the median as the cut-off value [12-14], resulting in groups of similar size. Next, we evaluated the influence of FAK in different molecular subtypes. Our results highlight the most pronounced prognostic relevance of FAK for DFS in $\mathrm{ER}^{+} \mathrm{HER} 2^{-} \mathrm{BC}$ and especially in luminal B-like tumors. In particular, for $\mathrm{ER}^{+} \mathrm{HER}^{-} \mathrm{BC}$, studies on prognostic factors are of utmost importance for a more precise differentiation between high- and low-risk patients with additional therapeutic options. There has been no clear consensus in publications about the relevance of FAK in various molecular subtypes. Alexopoulou et al. [14] showed a correlation between FAK expression and luminal $\mathrm{A}$ as well as triple-negative tumor cells. Conversely, Lark and co-workers [15] discussed a possible molecular interaction between FAK and HER2 in which HER2 regulates FAK phosphorylation. This hypothesis was followed by the findings of Schmitz et al. [16], who found no prognostic influence, concluding that this may have been due to the composition of their cohort. Like our study population, their cohort comprised patients with early tumor stages. Golubovskaya et al. [17] described contrasting correlations. However, most of the authors regarded the limited number of patients and the heterogeneity of the study cohorts as possible causes for the inconsistent results. The challenge of comparability also results from variations in the FAK detection (phosphorylation status, cell compartment, etc.).

Recently, first results of an ATP-competitive FAK inhibitor (PF-562271) were published and showed promising initial results [18]. Several FAK inhibitors are currently in clinical testing (PF-
562271, PF-04554878, GSK2256098, VS-4718, VS-6062, VS-6063, and BI853520) $[19,20]$. Based on our results, it is possible that the implementation of FAK inhibitors may be most effective in the cohort of luminal B BC, perhaps in combination with an endocrine therapy, as known for other combinations such as everolimus or palbociclib. In particular, in the context of overcoming endocrine resistance, the use of an FAK inhibitor would be a further therapeutic option. Further pre-clinical and clinical studies are needed to answer these questions.

All previous studies on the prognostic impact of FAK have included cohorts of patients treated with adjuvant therapies, which may have confounded the prognostic impact of this biomarker. A particular strength of our study is the selection of our cohort of patients. None of the patients with node-negative $\mathrm{BC}$ had received adjuvant systemic therapy, thus excluding this potential bias. A potential weakness of our study is that no differentiation of the activity state of FAK was performed. Such a differentiation analysis may provide further information on the prognostic role of FAK in various subtypes of BC. Another potential weakness of our study is the limited number of patients ( $\mathrm{n}=335)$, especially in the subgroup analyses.

In conclusion, our study showed that FAK protein expression was associated with DFS in univariate but not in multivariate analyses. Prognostic relevance was most pronounced in $\mathrm{ER}^{+} \mathrm{HER} 2^{-} \mathrm{BC}$ and especially in luminal B-like tumors. This might lead to a preferential investigation of FAK inhibitors in these patients.

\section{Disclosure Statement}

The authors declare that they have no competing interests.

\section{References}

1 Zhao J, Guan JL: Signal transduction by focal adhesion kinase in cancer. Cancer Metastasis Rev 2009;28:3549.

2 Luo M, Fan H, Nagy T, et al.: Mammary epithelial-specific ablation of the focal adhesion kinase suppresses mammary tumorigenesis by affecting mammary cancer stem/progenitor cells. Cancer Res 2009;69:466-474.

3 Agochiya M, Brunton VG, Owens DW, et al.: Increased dosage and amplification of the focal adhesion kinase gene in human cancer cells. Oncogene 1999;18: 5646-5653.

4 Owens LV, Xu L, Craven RJ, et al.: Overexpression of the focal adhesion kinase (p125FAK) in invasive human tumors. Cancer Res 1995;55:2752-2755.

5 Cance WG, Harris JE, Iacocca MV, et al.: Immunohistochemical analyses of focal adhesion kinase expression in benign and malignant human breast and colon tissues: Correlation with preinvasive and invasive phenotypes. Clin Cancer Res 2000;6:2417-2423.

6 Lightfoot HM Jr, Lark A, Livasy CA, et al.: Upregulation of focal adhesion kinase (FAK) expression in ductal carcinoma in situ (DCIS) is an early event in breast tumorigenesis. Breast Cancer Res Treat 2004;88:109-116.

7 van Miltenburg MH, van Nimwegen MJ, Tijdens I, et al.: Mammary gland-specific ablation of focal adhesion kinase reduces the incidence of p53-mediated mammary tumour formation. Br J Cancer 2014;110:27472755.
8 Schmidt M, Victor A, Bratzel D, et al.: Long-term outcome prediction by clinicopathological risk classification algorithms in node-negative breast cancer-comparison between Adjuvant!, St Gallen, and a novel risk algorithm used in the prospective randomized NodeNegative-Breast Cancer-3 (NNBC-3) trial. Ann Oncol 2009;20:258-264.

9 McShane LM, Altman DG, Sauerbrei W, et al.: REporting recommendations for tumor MARKer prognostic studies (REMARK). Breast Cancer Res Treat 2006;100: 229-235.

10 Remmele W, Stegner HE: Recommendation for uniform definition of an immunoreactive score (IRS) for immunohistochemical estrogen receptor detection (ER-ICA) in breast cancer tissue (in German). Pathologe 1987;8:138-140.

11 Madan R, Smolkin MB, Cocker R, et al.: Focal adhesion proteins as markers of malignant transformation and prognostic indicators in breast carcinoma. Hum Pathol 2006;37:9-15.

12 Yom CK, Noh DY, Kim WH, Kim HS: Clinical significance of high focal adhesion kinase gene copy number and overexpression in invasive breast cancer. Breast Cancer Res Treat 2011;128:647-655

13 Theocharis SE, Klijanienko JT, Padoy E, et al.: Focal adhesion kinase (FAK) immunocytochemical expression in breast ductal invasive carcinoma (DIC): Correlation with clinicopathological parameters and tumor proliferative capacity. Med Sci Monit 2009;15:BR221-226.
14 Alexopoulou AN, Ho-Yen CM, Papalazarou V, et al.: Tumour-associated endothelial-FAK correlated with molecular sub-type and prognostic factors in invasive breast cancer. BMC Cancer 2014;14:237.

15 Lark AL, Livasy CA, Dressler L, et al.: High focal adhesion kinase expression in invasive breast carcinomas is associated with an aggressive phenotype. Mod Pathol 2005;18:1289-1294.

16 Schmitz KJ, Grabellus F, Callies R, et al.: High expression of focal adhesion kinase (p125FAK) in node-negative breast cancer is related to overexpression of HER-2/neu and activated Akt kinase but does not predict outcome. Breast Cancer Res 2005; 7:R194-203.

17 Golubovskaya VM, Ylagan L, Miller A, et al.: High focal adhesion kinase expression in breast carcinoma is associated with lymphovascular invasion and triplenegative phenotype. BMC Cancer 2014;14:769.

18 Infante JR, Camidge DR, Mileshkin LR, et al.: Safety, pharmacokinetic, and pharmacodynamic phase I doseescalation trial of PF-00562271, an inhibitor of focal adhesion kinase, in advanced solid tumors. J Clin Oncol 2012;30:1527-1533

19 Schultze A, Fiedler W: Clinical importance and potential use of small molecule inhibitors of focal adhesion kinase. Anticancer Agents Med Chem 2011;11:593-599.

20 Roy-Luzarraga M, Hodivala-Dilke K: Molecular pathways: Endothelial cell FAK-a target for cancer treatment. Clin Cancer Res 2016;22:3718-3724. 